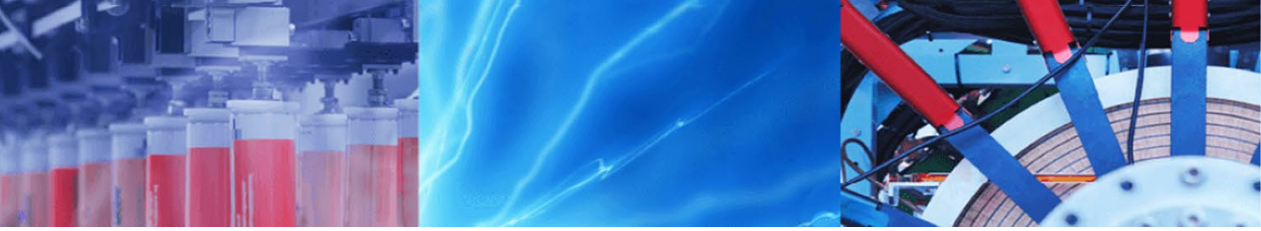

Research Article

\title{
16S rRNA gene sequencing and MALDI-TOF mass spectrometry based comparative assessment and bioprospection of psychrotolerant bacteria isolated from high altitudes under mountain ecosystem
}

\author{
Anita Pandey ${ }^{1} \cdot$ Rahul Jain ${ }^{1} \cdot$ Avinash Sharma $^{2} \cdot$ Kusum Dhakar $^{1} \cdot$ Gaurav S. Kaira $^{1} \cdot$ Praveen Rahi $^{2} \cdot$ Ashish Dhyani $^{1}$. \\ Neha Pandey ${ }^{1}$. Priyanka Adhikari ${ }^{1}$ - Yogesh S. Shouche ${ }^{2}$
}

(c) Springer Nature Switzerland AG 2019

\begin{abstract}
The Himalayan Mountains are placed among the globally recognized biodiversity hot spots. While the Indian Himalayan Region (IHR) has been subjected to extensive studies on plant and animal biodiversity, microbial diversity is now being studied for its bioprospection. The present paper deals with the evaluation of bacterial diversity in high-altitude soil samples from IHR following polyphasic approach including comparison between the MALDI-TOF mass spectrometry and 16S rRNA gene sequencing for species-level identification. Initially, a culture collection of large number of bacterial isolates was established in the laboratory. Performing morphological and biochemical screenings, sixty-one representative isolates were selected for mass spectrometry and gene sequencing. Both the methods emerged with bacterial identification showing maximum number of Bacillus followed by Pseudomonas species. The other frequently isolated strains belonged to the genera Alcaligenes, Carnobacterium, Lysinibacillus, Microbacterium, Paenarthrobacter, Rhodococcus, Serratia and Stenotrophomonas. Although the MALDI-TOF technique appeared to be advantageous as less time-consuming in comparison with 16S rRNA-based method, the discrepancies at species level indicated the limited database of MALDI Biotyper and species complexity in the genera. The remarkable characteristics of the bacterial isolates were their tolerance to wide range of $\mathrm{pH}$ and temperature. Their potential to produce industrially valuable enzymes indicated their importance in bioprospection. Accessioning of these bacterial isolates in microbial culture collections is a cautious effort for their availability to conduct advanced research on these cold-adapted bacteria in future.
\end{abstract}

Keywords Indian Himalaya · Bacterial diversity · Polyphasic approach · MALDI-TOF - 16S rRNA gene · Bioprospection

\section{Introduction}

Microorganisms can grow over a wide range of temperature. Accordingly, they have been divided into three broad categories-thermophiles, mesophiles, and psychrophiles. The last category is further subdivided into psychrophiles with optimal growth temperatures below $15^{\circ} \mathrm{C}$ and an upper limit of $20^{\circ} \mathrm{C}$, and psychrotrophs (psychrotolerants) with the ability to grow at $0{ }^{\circ} \mathrm{C}$ or below but grow optimally at temperatures around $20-25^{\circ} \mathrm{C}$ [27]. In the recent literature, the cold-adapted microorganisms have also been classified as stenopsychrophiles that grow in a narrow range of low temperature $\left(T_{\max } \sim 20^{\circ} \mathrm{C}\right.$ and $\left.T_{\text {opt }} 5-15^{\circ} \mathrm{C}\right)$ and eurypsychrophiles that can grow at the broad range of temperature (psychrotrophs/psychrotolerants; $T_{\text {max }}>20^{\circ} \mathrm{C}$ and $T_{\text {min }}<0^{\circ} \mathrm{C}$ with $T_{\text {opt }}>20^{\circ} \mathrm{C}$ ) [35]. Both psychrophiles and psychrotrophs are important in global ecology as the majority of aquatic and terrestrial ecosystems of our planet are permanently or seasonally submitted to low temperatures [14]. These microorganisms, therefore, should be regarded as the most

\footnotetext{
$\triangle$ Anita Pandey, anita@gbpihed.nic.in; anitapandey333@gmail.com | ${ }^{1}$ Centre for Environmental Assessment and Climate Change, G.B. Pant National Institute of Himalayan Environment and Sustainable Development, Kosi-Katarmal, Almora, Uttarakhand 263 643, India. ${ }^{2}$ National Centre for Microbial Resource, National Centre for Cell Science, Pune, Maharashtra 411021, India.
} 
successful colonizers of the planet Earth [36]. Psychrotolerant bacteria, along with their ability to adapt to the temperatures close to or below freezing, require specific strategies for the continuance of their metabolic activities including maintenance of the membrane fluidity and the protein synthesis at low temperature. Furthermore, the psychrotolerant bacteria and their cold-active enzymes are being focused for their importance in biotechnological applications and physiological adaptations $[1,2,26,41]$.

Accurate species-level identification is critical in view of understanding the biodiversity of a particular ecological niche. Species-level identification of cultured bacterial isolates usually relies on the polyphasic approach consisting of phenotypic and genotypic characters. Most of the genotypic identification methods are mainly based on the polymorphism of the $16 \mathrm{~S}$ rRNA gene sequences. Sequencing of the 16S rRNA gene has been accepted as the reference method for species-level identification of various groups of bacteria, for which the availability of an extensive and comprehensive quality-controlled database is a prerequisite [13]. In further evolution on the subject line, matrix-assisted laser desorption/ionization-time-of-flight mass spectrometry (MALDI-TOF MS) emerged as a promising technology for species-level identification. It may be a useful tool if a detailed database is available. In particular, this technique is being considered useful for clinical isolates where databases are quite satisfying. However, for environmental bacteria (particularly for extreme environments) lot of database upgrading is needed [45]. The technology involves analysis of protein composition of a bacterial cell by measuring the exact size of peptides and small proteins, which is assumed to be characteristic of each bacterial species. This technology has the merit to identify the strains within few minutes, starting the analysis with the whole cells, cell lysates, or crude bacterial extracts $[10,15,34]$.

Himalaya is one of the coldest regions on Earth and represents one of the 34 globally recognized biodiversity hot spots. Microbial diversity in high altitudes of Indian Himalayan Region (IHR) is increasingly receiving attention mainly for their bioprospection, conservation, and the associated survival strategies $[7,49]$. The present study aims to assess the culturable bacterial diversity from high altitudes of IHR on the basis of their phenotypic characters, the 16S rRNA gene sequencing, and the MALDI-TOF MS. The importance of this bacterial diversity in bioprospection is also emphasized.

\section{Materials and methods}

\subsection{Study sites}

The study area covered a wide range of altitudes, including the temperate, sub-alpine, and alpine zones of Pindari
Glacier region $\left(33^{\circ} 5^{\prime}-30^{\circ} 10^{\prime} \mathrm{N}\right.$ to $79^{\circ} 48^{\prime}-79^{\circ} 52^{\prime} \mathrm{E}$ ) and cold desert $\left(30^{\circ} 46^{\prime} 24.8^{\prime \prime} \mathrm{N} ; 79^{\circ} 29^{\prime} 33.4^{\prime \prime}\right)$, between 1800 and $3610 \mathrm{~m}$ above mean sea level in IHR. The area is identified with heavy rainfalls and snowfalls, maintaining low temperature up to sub-zero levels. The soil $\mathrm{pH}$ at the sampling sites ranged between 4.5 and 6.5 . The mean monthly temperature is reported to be between $5.5^{\circ} \mathrm{C}$ (January) and $21.5^{\circ} \mathrm{C}$ (August) [30].

\subsection{Isolation and characterization of bacteria}

Bacteria, based on the distinct colony morphology, were initially isolated from the soil dilution agar plates following incubation at different temperatures $\left(9,14,21\right.$ and $\left.28^{\circ} \mathrm{C}\right)$ on tryptone yeast extract (TY) and nutrient agar (NA) media using standard serial dilution method. A culture collection of pure bacterial isolates was established and maintained at $4{ }^{\circ} \mathrm{C}$ in a refrigerator and as glycerol stocks at $-20^{\circ} \mathrm{C}$, till further use. The large number of pure bacterial isolates (more than 500) obtained from the agar plates were de-replicated using multiple screening on the basis of growth characteristics, colony and cell morphology, and a number of biochemical characters. Finally, 61 bacterial isolates were selected for the present study.

Colony morphology of the bacterial isolates was recorded from the agar plates following incubation at $21^{\circ} \mathrm{C}$ for 2-5 days. Cell morphology was studied using a Nikon Eclipse 50i microscope in oil emersion. The growth requirements of the bacterial isolates were performed subject to different temperatures $\left(5-60^{\circ} \mathrm{C}\right.$ with an interval of $\left.5^{\circ}\right)$ and $\mathrm{pH}$ levels, i.e., $1.0-14.0$ (with an interval of $1.0 \mathrm{pH}$ ), for their minimum, optimum, and maximum temperature and $\mathrm{pH}$ range.

\subsection{Enzyme assays}

Bioassays for three enzymes (amylase, lipase, and protease) were performed using starch $(0.2 \%)$, tributyrin $(0.1 \%)$, and skim milk (3.0\%) in the medium, separately. The plates were incubated at $25^{\circ} \mathrm{C}$, and observations were recorded after $72 \mathrm{~h}$.

\subsection{MALDI-TOF MS}

Loopful taken from the fresh cultures of bacteria was harvested in $1.5-\mathrm{ml}$ vials containing $300 \mu$ l of deionized water and mixed thoroughly by vortexing for a minute. Absolute ethanol $(900 \mu \mathrm{l})$ was added and mixed thoroughly. The suspended cells were centrifuged at 10,000 rpm for $2 \mathrm{~min}$. Following centrifugation, supernatant was carefully discarded without disturbing the pellet. The pellet was airdried to get rid of the residual ethanol. Dried pellet was then re-suspended in $70 \%$ formic acid ( $20 \mu \mathrm{l})$ by vigorous 
mixing followed by the addition of acetonitrile $(20 \mu \mathrm{l})$ and mixed thoroughly. This mixture was again centrifuged at maximum speed $(13,000 \mathrm{rpm})$ so that all the material is collected neatly in a pellet. $1 \mu$ l of extract (clear supernatant) was placed on a MALDI standard target, air-dried, and overlaid with the $1 \mu$ l of alpha-cyano-4-hydroxycinnamic acid (HCCA) matrix; the target plate was allowed to dry at room temperature; and then it was loaded in the instrument AUTOFLEX TOF/TOF (Bruker Daltonik GmbH, Germany) [19].

Mass spectra were acquired in a linear positive ion extraction mode at a laser frequency of $200 \mathrm{~Hz}$, within a mass range from 2000 to $20,000 \mathrm{Da}$. The ion source 1 voltage was $19.5 \mathrm{kV}$, ion source 2 voltage was maintained at $18.2 \mathrm{kV}$, lens voltage was $7 \mathrm{kV}$, and the extraction delay time was $240 \mathrm{~ns}$. The spectra were calibrated externally using the standard calibrant mixture (Escherichia coli extracts including the additional proteins RNase $A$ and myoglobin, Bruker Daltonics). The MALDI Biotyper software 3.0 (Bruker Daltonik) was used to identify the bacterial isolates and to visualize the mass spectra. Species-level identity was considered for the isolates with biotyper score value $>2.0$, while the score value ranging from 1.7 to 1.99 was considered for genus-level identity.

\subsection{S rRNA gene sequencing}

The DNA extraction was performed using single colony enriched in TY agar. The 16S rDNA was amplified with bacterial specific primers 27F (5'-GAGTTTGATCMTGGCTC AG-3') and 1492R (5'-TACGGYTACCTTGTTACGACTT-3'). The PCR cycling conditions included initial denaturation at $94^{\circ} \mathrm{C}(3 \mathrm{~min})$, followed by 35 cycles of denaturation at $94^{\circ} \mathrm{C}(1 \mathrm{~min})$, annealing at $55^{\circ} \mathrm{C}(1 \mathrm{~min})$ and extension at $72{ }^{\circ} \mathrm{C}(1 \mathrm{~min})$, followed by final extension for a period of $10 \mathrm{~min}$ at $72{ }^{\circ} \mathrm{C}$. The amplified PCR product was purified with a mixture of $20 \%$ polyethylene glycol (PEG) and $2.5 \mathrm{M} \mathrm{NaCl}$. Sequencing was conducted using 96-capillary 3730xI DNA Analyzer (Hitachi). The identity of the isolates was determined through $16 \mathrm{~S}$ rRNA gene sequence analysis using EzTaxon database search. The sequences were aligned using MUSCLE algorithm [9]. Phylogenetic and molecular evolutionary analyses were conducted using MEGA, version 7.0 [20]. The phylogenetic tree was constructed by the neighbour joining method [37], using the distance matrix from the alignment.

\subsection{Accessioning of bacterial cultures and the nucleotide sequences}

The bacterial cultures and their nucleotide sequences have been accessioned at National Centre for Microbial Resource, National Centre for Cell Science, Pune, India [39] and at https://www.ncbi.nlm.nih.gov/, respectively; the accession numbers are given in Table 2.

\section{Results and discussion}

\subsection{Characteristics of psychrotolerant bacteria}

Almost all the bacterial isolates developed colonies on TY agar within 2-7 days of incubation at $25^{\circ} \mathrm{C}$. Compilation of the results on colony morphology, Gram staining, temperature and $\mathrm{pH}$ requirement, and enzyme activity with respect to amylase, lipase, and protease of 61 bacterial isolates is presented in Table 1. The bacterial isolates represented $38 \mathrm{Gram}$-positive and $23 \mathrm{Gram}$-negative species. The remarkable features of the bacteria were observed as their ability to grow at wide range of temperature and $\mathrm{pH}$. All the bacterial isolates, except 2 (GBPI_Hb9 and GBPI_ $\mathrm{Hb34}$ ), could grow at $5^{\circ} \mathrm{C}$. The upper limit tolerance for temperature was recorded at 35,45 , and $55^{\circ} \mathrm{C}$ in case of 24,25 , and 12 bacterial isolates, respectively. Two of the isolates could grow up to $15^{\circ} \mathrm{C}$ and one up to $20^{\circ} \mathrm{C}$ only. Similarly, the bacterial isolates could grow at wide range of $\mathrm{pH}$ (minimum 2-4 to maximum 12-14). The isolates grew luxuriantly between 5 and $9 \mathrm{pH}$.

Microorganisms from extreme environments are increasingly receiving recognition mainly due to their remarkable phenotypic characteristics, and one of such characteristics in the present study, is their ability to tolerate wide range of $\mathrm{pH}$ from extreme acidic to extreme alkaline. This phenomenon has recently been reviewed with reference to the bacteria and fungi that were isolated from extreme high-temperature (hot springs) as well as extreme low-temperature (cold deserts, glaciers, and temperate forests) environments. The majority of the case studies analysed in this review were from Indian Himalaya conducted over more than 20 years. Tolerance to wide $\mathrm{pH}$ range was considered as a hidden character in view of their isolation sites with neutral or slightly acidic/alkaline $\mathrm{pH}$. It was considered that the genome of these organisms is a probable reservoir of the hidden variations that result in the expression of the associated specific genes in response to environmental stress [7]. On the similar lines, tolerance to wide range of temperature is a prerequisite for the microorganisms that survive in an environment with frequent fluctuations in temperature. These features can be attributed to the strategies evolved in these microorganisms to adapt to the extreme environments. Tolerance of microorganisms to multiple environmental stresses is referred to as polyextremophile that is now emerging as an interesting topic in extreme environments research [38].

Besides the 61 bacterial isolates under consideration, a number of bacterial cultures were observed in VBNC 
Table 1 Characteristics of the bacterial isolates

\begin{tabular}{|c|c|c|c|c|c|c|c|c|}
\hline \multirow[t]{2}{*}{ S. No. } & \multirow[t]{2}{*}{ Isolate code } & \multirow[t]{2}{*}{ Colony morphology } & \multirow[t]{2}{*}{ Microscopy } & \multicolumn{2}{|c|}{$\begin{array}{l}\text { Temperature }\left({ }^{\circ} \mathrm{C}\right) \\
\text { and } \mathrm{pH} \text { limit }\end{array}$} & \multicolumn{3}{|c|}{ Enzyme activity } \\
\hline & & & & Temp & $\mathrm{pH}$ & Amy & Lip & Pro \\
\hline 1 & GBPI_Hb0 & Watery, slimy, raised, medium & Gram -ve rods & $\begin{array}{l}5-45 \\
(25)\end{array}$ & $\begin{array}{l}3-14 \\
(5-7)\end{array}$ & + & + & + \\
\hline 2 & GBPI_Hb1 & Round, smooth, flat, large & Gram -ve rods & $\begin{array}{l}5-55 \\
(25)\end{array}$ & $\begin{array}{l}4-14 \\
(5-7)\end{array}$ & + & + & + \\
\hline 3 & GBPI_Hb5 & Round, smooth, raised, small & Gram -ve rods & $\begin{array}{l}5-45 \\
(25)\end{array}$ & $\begin{array}{l}2-14 \\
(5-7)\end{array}$ & - & + & + \\
\hline 4 & GBPI_Hb6 & Round, smooth, small & Gram -ve rods & $\begin{array}{l}5-45 \\
(25)\end{array}$ & $\begin{array}{l}3-14 \\
(7-9)\end{array}$ & - & + & + \\
\hline 5 & GBPI_Hb7 & Spread, undulated, flat, medium & Gram + ve rods & $\begin{array}{l}5-35 \\
(25)\end{array}$ & $\begin{array}{l}4-14 \\
(5-7)\end{array}$ & + & + & + \\
\hline 6 & GBPI_Hb9 & Spread, irregular, flat, medium & Gram + ve rods & $\begin{array}{l}9-55 \\
(35)\end{array}$ & $\begin{array}{l}4-12 \\
(5-7)\end{array}$ & + & + & + \\
\hline 7 & GBPI_Hb12 & Round, undulated, flat, medium & Gram + ve rods & $\begin{array}{l}5-55 \\
(35)\end{array}$ & $\begin{array}{l}3-14 \\
(5-7)\end{array}$ & + & + & - \\
\hline 8 & GBPI_Hb14 & Round, smooth, flat, small & Gram + ve rods & $\begin{array}{l}5-45 \\
(25)\end{array}$ & $\begin{array}{l}2-14 \\
(5-9)\end{array}$ & - & + & - \\
\hline 9 & GBPI_Hb15 & Concentric, convex, smooth, large & Gram + ve rods & $\begin{array}{l}5-55 \\
(35)\end{array}$ & $\begin{array}{l}2-14 \\
5-9)\end{array}$ & + & + & + \\
\hline 10 & GBPI_Hb17 & Wrinkled, smooth, raised, large & Gram + ve rods & $\begin{array}{l}5-55 \\
(25)\end{array}$ & $\begin{array}{l}2-14 \\
(5-9)\end{array}$ & + & + & - \\
\hline 11 & GBPI_Hb18 & Round, smooth, small drops & Gram -ve rods & $\begin{array}{l}5-45 \\
(25)\end{array}$ & $\begin{array}{l}2-15 \\
(5-9)\end{array}$ & - & + & - \\
\hline 12 & GBPI_Hb19 & Round, smooth, medium drops & Gram -ve rods & $\begin{array}{l}5-45 \\
(25)\end{array}$ & $\begin{array}{l}2-14 \\
(5-9)\end{array}$ & - & + & - \\
\hline 13 & GBPI_Hb24 & Round, smooth, convex, large & Gram + ve rods & $\begin{array}{l}5-55 \\
(35)\end{array}$ & $\begin{array}{l}2-12 \\
(7-9)\end{array}$ & + & + & + \\
\hline 14 & GBPI_Hb34 & Round, smooth, small drops & Gram -ve rods & $\begin{array}{l}9-35 \\
(25)\end{array}$ & $\begin{array}{l}2-14 \\
(7-9)\end{array}$ & - & + & - \\
\hline 15 & GBPI_Hb37 & Rhizoidal, irregular, flat, large & Gram + ve rods & $\begin{array}{l}5-35 \\
(25)\end{array}$ & $\begin{array}{l}3-14 \\
(7-9)\end{array}$ & + & + & - \\
\hline 16 & GBPI_Hb41 & Rhizoidal, irregular, flat, large & Gram + ve rods & $\begin{array}{l}5-35 \\
(25)\end{array}$ & $\begin{array}{l}3-14 \\
(5-7)\end{array}$ & - & + & + \\
\hline 17 & GBPI_Hb52 & Round, smooth, convex, small & Gram + ve rods & $\begin{array}{l}5-35 \\
(25)\end{array}$ & $\begin{array}{l}2-14 \\
(7-9)\end{array}$ & - & + & - \\
\hline 18 & GBPI_Hb55 & Rhizoidal, flat, irregular, large & Gram + ve rods & $\begin{array}{l}5-35 \\
(25)\end{array}$ & $\begin{array}{l}4-14 \\
(7-9)\end{array}$ & + & + & + \\
\hline 19 & GBPI_Hb61 & Round, smooth, flat, small & Gram -ve rods & $\begin{array}{l}5-35 \\
(25)\end{array}$ & $\begin{array}{l}4-14 \\
(5-7)\end{array}$ & - & + & + \\
\hline 20 & GBPI_Hb120 & Round, smooth, medium drops & Gram -ve rods & $\begin{array}{l}5-35 \\
(25)\end{array}$ & $\begin{array}{l}2-14 \\
(5-9)\end{array}$ & - & + & - \\
\hline 21 & GBPI_Hb149 & Wrinkled, raised, undulated, medium & Gram -ve rods & $\begin{array}{l}5-35 \\
(25)\end{array}$ & $\begin{array}{l}2-14 \\
(5-7)\end{array}$ & - & + & - \\
\hline 22 & GBPI_Hb171 & Round, smooth, small drops & Gram -ve rods & $\begin{array}{l}5-45 \\
(25)\end{array}$ & $\begin{array}{l}3-14 \\
(7-9)\end{array}$ & - & + & + \\
\hline 23 & GBPI_Hb227 & Round, smooth, small drops & Gram -ve rods & $\begin{array}{l}5-35 \\
(25)\end{array}$ & $\begin{array}{l}2-14 \\
(5-7)\end{array}$ & - & + & - \\
\hline 24 & GBPI_Hb232 & Rhizoidal, flat, irregular, large & Gram + ve rods & $\begin{array}{l}5-35 \\
(25)\end{array}$ & $\begin{array}{l}4-14 \\
(7-9)\end{array}$ & + & + & + \\
\hline 25 & GBPI_Hb240 & Spread, irregular, flat, small & Gram + ve rods & $\begin{array}{l}5-45 \\
(25)\end{array}$ & $\begin{array}{l}2-14 \\
(5-9)\end{array}$ & + & + & + \\
\hline 26 & GBPI_Hb243 & Round, smooth, flat, large & Gram + ve rods & $\begin{array}{l}5-45 \\
(35)\end{array}$ & $\begin{array}{l}3-14 \\
(5-7)\end{array}$ & + & + & + \\
\hline
\end{tabular}


Table 1 (continued)

\begin{tabular}{|c|c|c|c|c|c|c|c|c|}
\hline \multirow[t]{2}{*}{ S. No. } & \multirow[t]{2}{*}{ Isolate code } & \multirow[t]{2}{*}{ Colony morphology } & \multirow[t]{2}{*}{ Microscopy } & \multicolumn{2}{|c|}{$\begin{array}{l}\text { Temperature }\left({ }^{\circ} \mathrm{C}\right) \\
\text { and } \mathrm{pH} \text { limit }\end{array}$} & \multicolumn{3}{|c|}{ Enzyme activity } \\
\hline & & & & Temp & $\mathrm{pH}$ & Amy & Lip & Pro \\
\hline 27 & GBPI_Hb249 & Round, undulated, raised, large & Gram +ve rods & $\begin{array}{l}5-55 \\
(35)\end{array}$ & $\begin{array}{l}3-12 \\
(5-7)\end{array}$ & + & + & + \\
\hline 28 & GBPI_Hb256 & Round, smooth, raised, medium & Gram +ve rods & $\begin{array}{l}5-45 \\
(35)\end{array}$ & $\begin{array}{l}2-14 \\
(5-7)\end{array}$ & + & + & + \\
\hline 29 & GBPI_Hb308 & Round, smooth, raised, large & Gram +ve rods & $\begin{array}{l}5-55 \\
(35)\end{array}$ & $\begin{array}{l}3-14 \\
(5-7))\end{array}$ & + & + & + \\
\hline 30 & GBPI_Hb309 & Round, smooth, small & Gram +ve rods & $\begin{array}{l}5-45 \\
(15)\end{array}$ & $\begin{array}{l}3-14 \\
(5-9)\end{array}$ & - & + & - \\
\hline 31 & GBPI_Hb310 & Round, smooth, umbonate, medium & Gram +ve rods & $\begin{array}{l}5-55 \\
(35)\end{array}$ & $\begin{array}{l}2-14 \\
(5-7)\end{array}$ & - & + & + \\
\hline 32 & GBPI_Hb312 & Rhizoidal, irregular, flat, large & Gram + ve rods & $\begin{array}{l}5-35 \\
(25)\end{array}$ & $\begin{array}{l}2-14 \\
(7-9)\end{array}$ & + & + & + \\
\hline 33 & GBPI_Hb314 & Round, smooth, convex, medium & Gram -ve rods & $\begin{array}{l}5-45 \\
(35)\end{array}$ & $\begin{array}{l}3-14 \\
(7-9)\end{array}$ & + & + & + \\
\hline 34 & GBPI_Hb330 & Spread, smooth, flat, large & Gram +ve rods & $\begin{array}{l}5-45 \\
(35)\end{array}$ & $\begin{array}{l}2-14 \\
(5-9)\end{array}$ & + & + & + \\
\hline 35 & GBPI_Hb501 & Round, smooth, small & Gram +ve rods & $\begin{array}{l}5-45 \\
(15)\end{array}$ & $\begin{array}{l}3-14 \\
(5-9)\end{array}$ & - & + & - \\
\hline 36 & GBPI_CDB5 & Round, smooth, flat, medium & Gram -ve rods & $\begin{array}{l}5-35 \\
(25)\end{array}$ & $\begin{array}{l}3-14 \\
(5-7)\end{array}$ & - & + & - \\
\hline 37 & GBPI_CDB6 & Round, smooth, raised, medium & Gram + ve rods & $\begin{array}{l}5-45 \\
(25)\end{array}$ & $\begin{array}{l}4-14 \\
(7-9)\end{array}$ & - & - & - \\
\hline 38 & GBPI_CDB51 & Round, smooth, small drops & Gram +ve rods & $\begin{array}{l}5-55 \\
(25)\end{array}$ & $\begin{array}{l}4-12 \\
(7-9)\end{array}$ & + & + & + \\
\hline 39 & GBPI_CDB57 & Round, undulated, raised, medium & Gram +ve rods & $\begin{array}{l}5-35 \\
(25)\end{array}$ & $\begin{array}{l}4-14 \\
(7-9)\end{array}$ & + & + & + \\
\hline 40 & GBPI_CDB58 & Round, smooth, raised, medium & Gram + ve rods & $\begin{array}{l}5-45 \\
(25)\end{array}$ & $\begin{array}{l}3-14 \\
(5-7)\end{array}$ & + & + & + \\
\hline 41 & GBPI_CDB61 & Round, smooth, medium drops & Gram + ve rods & $\begin{array}{l}5-45 \\
(35)\end{array}$ & $\begin{array}{l}3-14 \\
(7-9)\end{array}$ & + & + & + \\
\hline 42 & GBPI_CDB65 & Round, smooth, raised, medium & Gram +ve rods & $\begin{array}{l}5-35 \\
(25)\end{array}$ & $\begin{array}{l}3-14 \\
(7-9)\end{array}$ & + & + & + \\
\hline 43 & GBPI_CDB76 & Concentric, flat, smooth, large & Gram +ve rods & $\begin{array}{l}5-55 \\
(35)\end{array}$ & $\begin{array}{l}3-14 \\
(5-7)\end{array}$ & + & + & + \\
\hline 44 & GBPI_CDB77 & Round, smooth, drop, medium & Gram -ve rods & $\begin{array}{l}5-35 \\
(25)\end{array}$ & $\begin{array}{l}4-14 \\
(7-9)\end{array}$ & - & + & + \\
\hline 45 & GBPI_CDB84 & Round, smooth, medium drops & Gram -ve rods & $\begin{array}{l}5-35 \\
(25)\end{array}$ & $\begin{array}{l}2-14 \\
(7-9)\end{array}$ & - & + & + \\
\hline 46 & GBPI_CDB86 & Round, smooth, medium drops & Gram + ve rods & $\begin{array}{l}5-35 \\
(25)\end{array}$ & $\begin{array}{l}4-14 \\
(7-9)\end{array}$ & + & + & - \\
\hline 47 & GBPI_CDB87 & Round, smooth, small & Gram -ve rods & $\begin{array}{l}5-45 \\
(25)\end{array}$ & $\begin{array}{l}2-14 \\
(5-7)\end{array}$ & - & + & + \\
\hline 48 & GBPI_CDB94 & Round, smooth, medium drops & Gram -ve rods & $\begin{array}{l}5-35 \\
(25)\end{array}$ & $\begin{array}{l}3-14 \\
(7-9)\end{array}$ & - & + & - \\
\hline 49 & GBPI_CDB96 & Round, smooth, medium drops & Gram + ve rods & $\begin{array}{l}5-35 \\
(25)\end{array}$ & $\begin{array}{l}3-14 \\
(5-7)\end{array}$ & + & - & - \\
\hline 50 & GBPI_CDB140 & Round, smooth, raised, medium & Gram + ve rods & $\begin{array}{l}5-55 \\
(35)\end{array}$ & $\begin{array}{l}3-14 \\
(5-9)\end{array}$ & + & + & + \\
\hline 51 & GBPI_CDB141 & Wrinkled, raised undulated, medium & Gram + ve rods & $\begin{array}{l}5-35 \\
(25)\end{array}$ & $\begin{array}{l}3-12 \\
(5-7)\end{array}$ & + & + & + \\
\hline 52 & GBPI_CDB142 & Concentric, undulated, raised & Gram + ve rods & $\begin{array}{l}5-45 \\
(25)\end{array}$ & $\begin{array}{l}2-14 \\
(5-9)\end{array}$ & + & + & - \\
\hline
\end{tabular}


Table 1 (continued)

\begin{tabular}{|c|c|c|c|c|c|c|c|c|}
\hline \multirow[t]{2}{*}{ S. No. } & \multirow[t]{2}{*}{ Isolate code } & \multirow[t]{2}{*}{ Colony morphology } & \multirow[t]{2}{*}{ Microscopy } & \multicolumn{2}{|c|}{$\begin{array}{l}\text { Temperature }\left({ }^{\circ} \mathrm{C}\right) \\
\text { and } \mathrm{pH} \text { limit }\end{array}$} & \multicolumn{3}{|c|}{ Enzyme activity } \\
\hline & & & & Temp & $\mathrm{pH}$ & Amy & Lip & Pro \\
\hline 53 & GBPI_CDB143 & Round, smooth, small drops & Gram -ve rods & $\begin{array}{l}5-35 \\
(25)\end{array}$ & $\begin{array}{l}2-14 \\
(5-9)\end{array}$ & - & + & - \\
\hline 54 & GBPI_CDB144 & Round, smooth, raised, medium & Gram +ve rods & $\begin{array}{l}5-45 \\
(25)\end{array}$ & $\begin{array}{l}4-12 \\
(5-7)\end{array}$ & - & + & - \\
\hline 55 & GBPI_CDB145 & Round, smooth, raised, medium & Gram +ve rods & $\begin{array}{l}5-45 \\
(35)\end{array}$ & $\begin{array}{l}2-14 \\
(5-9)\end{array}$ & - & + & - \\
\hline 56 & GBPI_CDB146 & Concentric, flat, undulated, large & Gram +ve rods & $\begin{array}{l}5-35 \\
(25)\end{array}$ & $\begin{array}{l}3-14 \\
(7-9)\end{array}$ & - & + & - \\
\hline 57 & GBPI_CDB147 & Round, smooth, medium drops & Gram -ve rods & $\begin{array}{l}5-45 \\
(25)\end{array}$ & $\begin{array}{l}2-14 \\
(5-9)\end{array}$ & - & - & - \\
\hline 58 & GBPI_CDB149 & Round, smooth, raised, small & Gram -ve rods & $\begin{array}{l}5-35 \\
(20)\end{array}$ & $\begin{array}{l}2-14 \\
(5-8)\end{array}$ & - & + & + \\
\hline 59 & GBPI_506 & Round, smooth, raised, medium & Gram -ve rods & $\begin{array}{l}5-45 \\
(25)\end{array}$ & $\begin{array}{l}2-14 \\
(5-8)\end{array}$ & - & + & - \\
\hline 60 & GBPI_507 & Round, smooth, raised, medium & Gram -ve rods & $\begin{array}{l}5-45 \\
(25)\end{array}$ & $\begin{array}{l}2-14 \\
(5-8)\end{array}$ & - & + & + \\
\hline 61 & GBPI_508 & Round, smooth, convex, medium & Gram -ve rods & $\begin{array}{l}5-45 \\
(25)\end{array}$ & $\begin{array}{l}2-14 \\
(5-8)\end{array}$ & - & + & + \\
\hline
\end{tabular}

Values in parentheses show the optimum temperature and $\mathrm{pH}$ requirement of the bacterial isolates; Amy. amylase; Lip. lipase; Pro. protease

(viable but not culturable) state. VBNC state has been attributed to the survival strategies possessed by the microorganisms against environmental stress such as low or high temperature [4]. Characteristics and resuscitation mechanisms of these microorganisms with respect to their survival under extreme conditions are now receiving attention in applied research [51].

\subsection{Comparative assessment of psychrotolerant bacteria using 16S rRNA gene sequencing and MALDI-TOF-based biotyping}

Quick and reliable methods are required to identify the unexplored diversity of any ecological niche. In this study, matrix-assisted laser desorption/ionization-time-of-flight mass spectrometry (MALDI-TOF MS)-based identification was included and the results were compared with $16 \mathrm{~S}$ rRNA-based method of identification (Table 2). On the basis of 16S rRNA analysis, the bacteria represented 11 genera and 31 species. Among these Bacillus represented 13; Pseudomonas 7; Serratia, Rhodococcus and Lysinibacillus 2 each; and Stenotrophomonas, Alcaligenes, Carnobacterium, Microbacterium and Paenarthrobacter 1 each (Fig. 1). The phylogenetic tree of the bacterial isolates along with their closely related species is presented in Fig. 2. MALDITOF MS-based biotyping showed similar identification of $3(4.92 \%)$ bacteria at species level and $36(59.01 \%)$ bacteria at genus level with respect to $16 \mathrm{~S}$ rRNA gene sequence analysis (Table 3). The discrepancies in identification were $54.10 \%$ and $13.11 \%$ at species and genus levels, respectively. Twelve (19.67\%) bacterial isolates could not be identified due to non-reliability in identification (log score $<1.7)$. Although the technique was less timeconsuming in comparison with $16 \mathrm{~S}$ rRNA-based method, the discrepancies at species level indicated limited reference database (main spectra, MSPs) of MALDI Biotyper for identification of soil microorganisms using MALDI-TOF MS technique.

The recent advances of new molecular technologies in genomics and proteomics offer alternatives to conventional microbiological procedures with respect to the identification of microorganisms. Advantages of polyphasic taxonomic approach that includes molecular identification techniques have been recognized and reviewed [5]. Koubek and co-workers [19] compared the three methods of taxonomic identification including biochemical methods, MALDI-TOF MS, and sequencing of 165 rRNA genes. Strejcek et al. [44] also compared whole-cell MALDI-TOF MS with 16S rRNA gene analysis for identification of recurrent bacterial isolates. The authors confirmed the potential of MALDI-TOF MS as a rapid screening method in environmental microbiology for determining similarities between bacterial isolates at genus level with various limitations such as low number of peaks, availability of reference mass spectra of only well-studied and described strains in database, etc. Therefore, agreement between MALDITOF MS-based biotyping and 16S rRNA gene sequencing was observed to be limited. While the emerging MALDI 
Table 2 Comparison of bacterial identification using 16S rRNA gene sequencing and MALDI-TOF MS-based biotyping

\begin{tabular}{|c|c|c|c|}
\hline S No. & Isolate (culture accession) ${ }^{\mathrm{a}}$ & 16S rRNA identification (\% similarity) (nucleotide accession) ${ }^{b}$ & Identification by MALDI (log score) \\
\hline 1 & GBPI_Hb0 (MCC 3300) & Pseudomonas granadensis (99.79) (HG764746) & Pseudomonas koreensis (2.2) \\
\hline 2 & GBPI_Hb1 & Serratia quinivorans (99.87) (AJ233430) & $\mathrm{NRI}$ \\
\hline 3 & GBPI_Hb5 (MCC 3295) & Pseudomonas paralactis (99.79) (AJ936933) & Pseudomonas fluorescens (2.1) \\
\hline 4 & GBPI_Hb6 & Stenotrophomonas maltophilia (100) & Stenotrophomonas maltophilia (2.2) \\
\hline 5 & GBPI_Hb7 & Bacillus wiedmannii (100) (AE016877) & Bacillus thuringiensis $(1.8)^{\mathrm{C}}$ \\
\hline 6 & GBPI_Hb9 (MCC 3123) & Bacillus velezensis (99.93) (JTKJ01000077) & $\mathrm{NRI}$ \\
\hline 7 & GBPI_Hb12 (MCC 3148) & Bacillus tequilensis (99.93) (AMXN01000021) & Bacillus subtilis (2.1) \\
\hline 8 & GBPI_Hb14 (MCC 3296) & Pseudomonas azotoformans (99.79) (AF074384) & Pseudomonas cedrina (2.0) \\
\hline 9 & GBPI_Hb15 & Bacillus mobilis (100) (ACNF01000156) & Bacillus cereus (2.2) \\
\hline 10 & GBPI_Hb17 (MCC3124) & Bacillus megaterium (99.93) (JJMH01000057) & NRI \\
\hline 11 & GBPI_Hb18 & Rhodococcus qingshengii (100) (X79289) & Rhodococcus erythropolis (2.5) \\
\hline 12 & GBPI_Hb19 & Serratia marcescens subsp. sakuensis (100) (JMPQ01000005) & ND \\
\hline 13 & GBPI_Hb24 & Bacillus aryabhattai (100) (EF114313) & Bacillus megaterium (1.8) \\
\hline 14 & GBPI_Hb34 (MCC3184) & Alcaligenes pakistanensis (99.48) (AJ242986) & Alcaligenes faecalis (1.8) \\
\hline 15 & GBPI_Hb37 & Bacillus weihenstephanensis (99.56) (AB049195) & Bacillus mycoides (2.1) \\
\hline 16 & GBPI_Hb41 & Bacillus weihenstephanensis (99.67) (ACMU01000002) & Pseudomonas extremorientalis (1.7) \\
\hline 17 & GBPI_Hb52 & Bacillus wiedmannii (100) (СР006863) & Lactobacillus sp (2.4) \\
\hline 18 & GBPI_Hb55 & Bacillus weihenstephanensis (99.90) (BAUY01000093) & Bacillus mycoides (2.0) \\
\hline 19 & GBPI_Hb61 (MCC 3297) & Pseudomonas proteolytica (99.30) (AJ537603) & Pseudomonas marginalis (2.0) \\
\hline 20 & GBPI_Hb120 (MCC3340) & Rhodococcus globerulus (100) (X80619) & Rhodococcus erythropolis (1.9) \\
\hline 21 & GBPI_Hb149 (MCC 3293) & Serratia marcescens subsp. sakuensis (99.93) (JMPQ01000005) & NRI \\
\hline 22 & GBPI_Hb171 (MCC 3143) & Stenotrophomonas maltophilia (100) (JALV01000036) & Stenotrophomonas maltophilia (2.3) \\
\hline 23 & GBPI_Hb227 & Rhodococcus qingshengii (100) (DQ185597) & Rhodococcus erythropolis (2.4) \\
\hline 24 & GBPI_Hb232 & Bacillus weihenstephanensis (100) (AB190217) & Bacillus mycoides (2.1) \\
\hline 25 & GBPI_Hb240 (MCC3317) & Bacillus mobilis (99.69) (ACNF01000156) & Bacillus thuringiensis (1.9) \\
\hline 26 & GBPI_Hb243 & Bacillus wiedmannii (99.63) (AE016877) & Bacillus cereus (1.8) \\
\hline 27 & GBPI_Hb249 (MCC3125) & Bacillus velezensis (99.79) (CP000560) & Bacillus cereus (2.1) \\
\hline 28 & GBPI_Hb256 & Bacillus cereus (100) (AE016877) & ND \\
\hline 29 & GBPI_Hb308 & Bacillus velezensis (99.37) (JTKJ01000077) & $\mathrm{NRI}$ \\
\hline 30 & GBPI_Hb309 & Carnobacterium maltaromaticum (99.92) (ND) & Lactobacillus sp (2.5) \\
\hline 31 & GBPI_Hb310 (MCC3140) & Bacillus safensis (100) (ASJD01000027) & NRI \\
\hline 32 & GBPI_Hb312 (MCC 3146) & Bacillus weihenstephanensis (100) (ACMU01000002) & NRI \\
\hline 33 & GBPI_Hb314 & Serratia marcescens subsp. sakuensis (99.93) (JPUX01000001) & Serratia marcescens (1.8) \\
\hline 34 & GBPI_Hb330 (MCC 3147) & Bacillus wiedmannii (99.93) (AE016877) & NRI \\
\hline 35 & GBPI_Hb501 (MCC3181) & Carnobacterium maltaromaticum (99.65) (JQMX01000001) & Lactobacillus sp (1.8) \\
\hline 36 & GBPI_CDB5 & Microbacterium maritypicum (99.76) (Y17227) & Microbacterium luteolum (1.9) \\
\hline 37 & GBPI_CDB6 & Bacillus paramycoides (99.88) (AB190217) & NRI \\
\hline 38 & GBPI_CDB51 (MCC 3149) & Bacillus atrophaeus (99.93) (AB021181) & Bacillus atrophaeus (2.0) \\
\hline 39 & GBPI_CDB57 & Bacillus atrophaeus (99.86) (AB021181) & ND \\
\hline 40 & GBPI_CDB58 & Brevibacterium frigoritolerans (99.91) (AM747813) & Bacillus atrophaeus (1.8) \\
\hline 41 & GBPI_CDB61 & Bacillus atrophaeus (99.64) (AB021181) & Bacillus atrophaeus (1.9) \\
\hline 42 & GBPI_CDB65 & Bacillus velezensis (99.79) (JTKJ01000077) & NRI \\
\hline 43 & GBPI_CDB76 & Bacillus siamensis (99.41) (JTKJ01000077) & Bacillus subtilis (1.7) \\
\hline 44 & GBPI_CDB77 & Rhodococcus qingshengii (99.33) (AP008957) & ND \\
\hline 45 & GBPI_CDB84 (MCC3341) & Pseudomonas paralactis (99.57) (AF074384) & Pseudomonas tolaasii (2.0) \\
\hline 46 & GBPI_CDB86 & Paenarthrobacter nitroguajacolicus (99.92) (AJ512504) & Arthrobacter aurescens (2.0) \\
\hline 47 & GBPI_CDB87 & Pseudomonas azotoformans (99.66) (ND) & Pseudomonas libanensis (2.1) \\
\hline 48 & GBPI_CDB94 & Serratia marcescens subsp. sakuensis (100) (JPUX01000001) & Arthrobacter aurescens (2.0) \\
\hline 49 & GBPI_CDB96 & Paenarthrobacter nitroguajacolicus (99.91) (AJ512504) & Arthrobacter nicotinovorans (1.9) \\
\hline 50 & GBPI_CDB140 & Bacillus atrophaeus (99.88) (AB021181) & Bacillus atrophaeus (2.0) \\
\hline
\end{tabular}


Table 2 (continued)

\begin{tabular}{llll}
\hline S No. & Isolate (culture accession) $^{\text {a }}$ & 16S rRNA identification (\% similarity) (nucleotide accession) & Identification by MALDI (log score) \\
\hline 51 & GBPI_CDB141 (MCC 3150) & Lysinibacillus xylanilyticus (99.66) (FJ477040) & NRI \\
52 & GBPI_CDB142 (MCC 3292) & Bacillus simplex (99.92) (ACNF01000156) & ND \\
53 & GBPI_CDB143 (MCC 3298) & Pseudomonas paralactis (99.93) (D84009) & Pseudomonas marginalis (2.0) \\
54 & GBPI_CDB144 & Bacillus simplex (99.92) (AB363738) & Bacillus muralis (1.8) \\
55 & GBPI_CDB145 (MCC 3166) & Lysinibacillus fusiformis (99.71) (AB271743) & Lysinibacillus fusiformis (1.8) \\
56 & GBPI_CDB146 & Bacillus weihenstephanensis (99.88) (ACMU01000002) & Bacillus mycoides (2.0) \\
57 & GBPI_CDB147 & Serratia marcescens subsp. sakuensis (100) (JPUX01000001) & Arthrobacter histidinolovorans (1.9) \\
58 & GBPI_CDB149 (MCC3294) & Pseudomonas marginalis (99.77) (ND) & NRI \\
59 & GBPI_506 (MCC2694) & Pseudomonas azotoformans (99.86) (D84009) & Pseudomonas extremorientalis (1.8) \\
60 & GBPI_507 (MCC2693) & Pseudomonas chlororaphis subsp. aurantiaca (100) (DQ682655) & Pseudomonas chlororaphis (2.1) \\
61 & GBPI_508 (MCC2692) & Pseudomonas palleroniana (99.72) (AY091527) & Pseudomonas veronii (1.8) \\
\hline
\end{tabular}

$N R I$ no reliable identification (log score $<1.7$ ); ND not done

${ }^{a}$ Culture accession of bacteria (in parentheses) deposited in National Centre for Microbial Resource, National Centre for Cell Science, Pune, India

${ }^{\mathrm{b}}$ Nucleotide accession of bacteria deposited in NCBI (second parentheses)

'Biotyper score values from 1.7 to 1.99 indicate genus-level identification

Fig. 1 Distribution of 61 bacterial isolates among 11 different bacterial genera

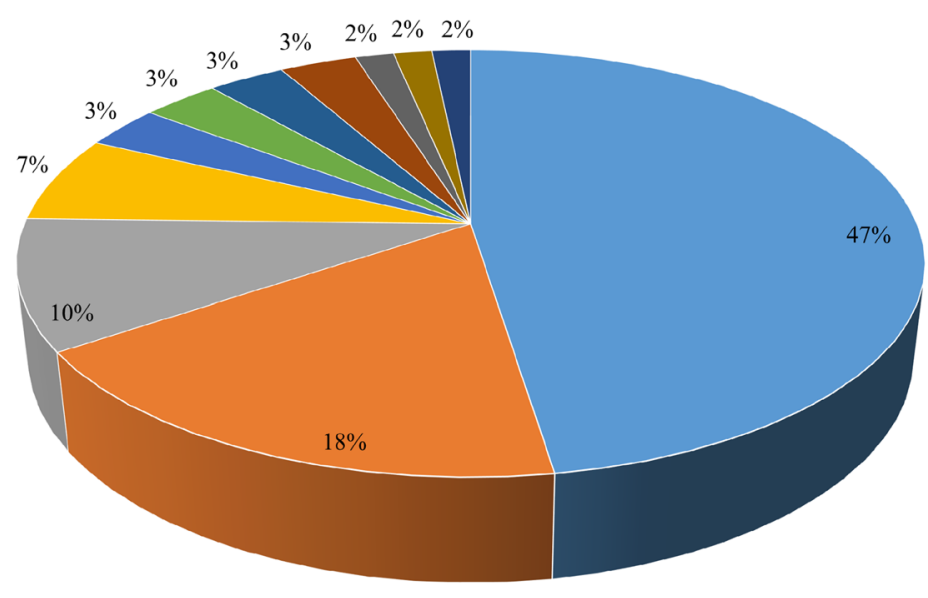

- Bacillus spp.

- Pseudomonas spp.

- Serratia spp.

- Rhodococcus spp.

- Lysinibacillus spp.

- S. maltophila

- P. nitroguajacolicus

- C. maltaromaticum

- B. frigoritolerans

- A. pakistanensis

- M. maritypicum biotyping is being popular in different fields of microbiology that has partly replaced conventional biochemical identification methods [12], variability of this methodology for the identification of microbes has been attributed to factors such as sample processing methods, matrix solutions, spectral quality, and data analysis methods [6, 21]. The methodology is becoming more popular in clinical set-ups for speedy and low-cost microbial identification $[28,34]$ and also in the analysis of bioactive compounds [46].

\subsection{Bioprospection of psychrotolerant bacteria}

Diversity of psychrotolerants in alpine cold habitats has already become a topic of interest in research with respect to their adaptation strategies and bioprospection [11, 23, $40,50]$. The present study showed predominance of the genus Bacillus which can be attributed to their spore-forming characteristic that allows them to survive under varied environmental extremes such as temperature, $\mathrm{pH}$, pressure [25]. Predominance of endospore formers is important due to their contribution in nutrient cycling and biodegradation. Pseudomonas was the second most dominating genus representing psychrotolerant culturable species. The genus Pseudomonas has been reported to inhabit the lowtemperature environments [29, 33]. Species of Bacillus and Pseudomonas have also been found suitable for developing the cold-tolerant bioinoculants for field applications under colder regions in IHR and Tropical Andes due to their plant growth-promoting and biocontrol abilities [31, 32]. The other

\section{SN Applied Sciences}


Fig. 2 Neighbour joining phylogenetic tree (bootstrap $=1000$ ) of representative bacterial isolates. The evolutionary distances were computed using the maximum composite likelihood method

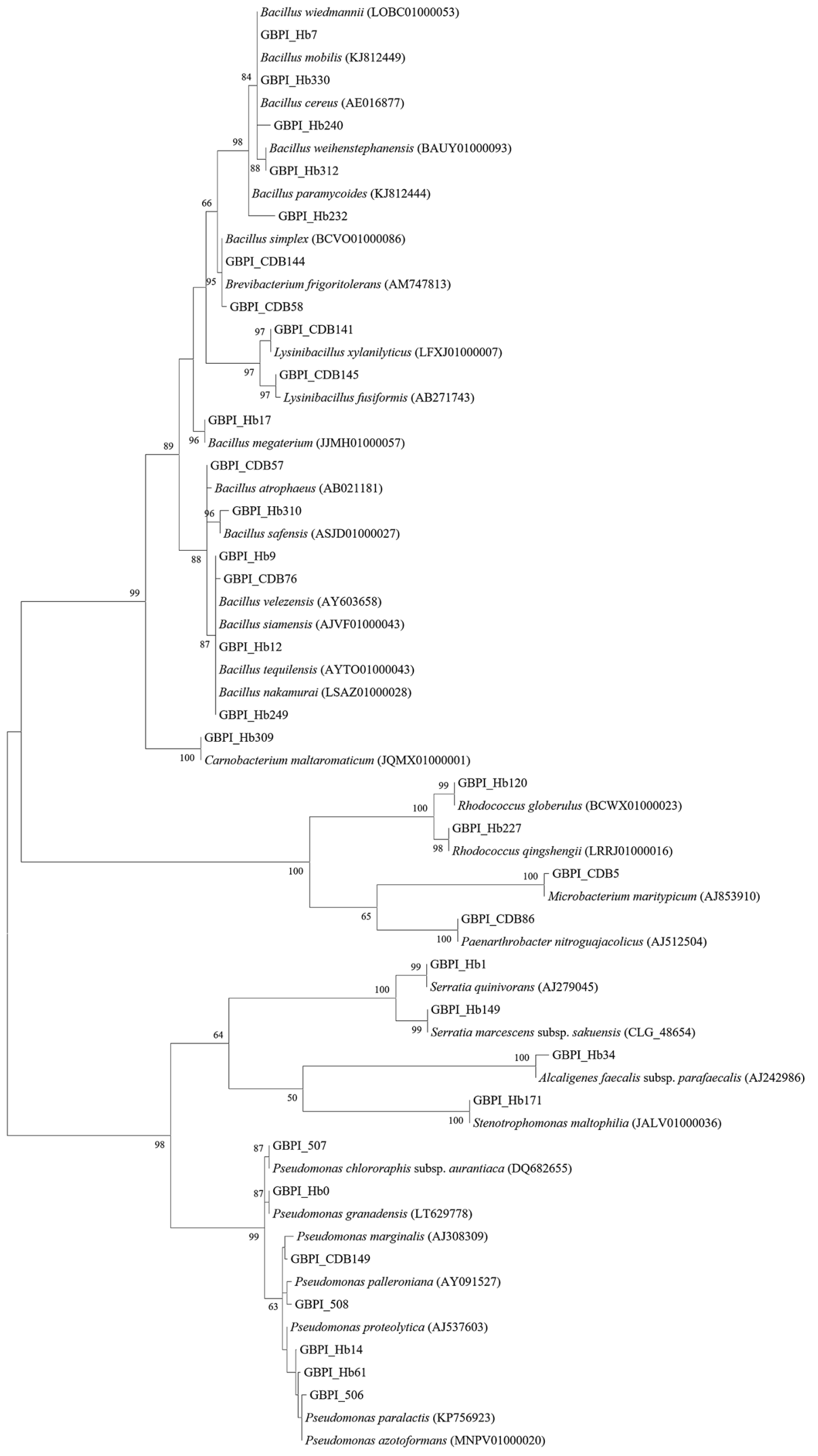


Table 3 Similarities and discrepancies in identification of 61 bacterial isolates identified by $16 \mathrm{~S}$ rRNA gene sequencing and MALDI-TOF MS technique

\begin{tabular}{llllll}
\hline Identification level & $\begin{array}{l}\text { 16S Identification by } \\
\text { rRNA (no. of isolates) }\end{array}$ & \multicolumn{2}{l}{$\begin{array}{l}\text { No. of isolates (\%) following MALDI-TOF MS-based } \\
\text { identification }\end{array}$} \\
\cline { 3 - 6 } & & Similarities & Discrepancies & NRI & ND \\
\hline Species level & 61 & $3(4.92)$ & $33(54.10)$ & $12(19.67)$ & $5(8.19)$ \\
Genus level & - & $36(59.01)$ & $8(13.11)$ & & \\
\hline
\end{tabular}

bacterial species belonging to Carnobacterium, Lysinibacillus, Microbacterium, Paenarthrobacter, Rhodococcus, Serratia and Stenotrophomonas have been known for their influences in ecological processes like biodegradation and bioremediation $[18,22,47]$. Some of these have also been reported as pathogens and of clinical importance $[3,48]$.

Out of sixty-one bacterial isolates, fifty-eight were positive for lipase, thirty-six for protease, and thirty for amylase. Twenty-two isolates were positive for all the three enzymes under consideration. While the enzymes produced by cold-adapted microorganisms are considered to play an important role in the survival of these organisms under temperature stress, they are also making their way to biotechnological industries with economic advantages $[2,8]$. Among the bacteria under study, Pseudomonas proteolytica has been reported to produce cold-active lipase [17]. Production of phenazine-1-carboxylic acid and laccase enzyme, from psychrotolerant and pigmented strains of Pseudomonas chlororaphis and Serratia marcescens, respectively, from this bacterial culture collection are few other examples in this line $[16,18]$. The ability of cold-tolerant microorganisms to grow and multiply at low temperatures is subjected to the adaptive changes in cellular proteins and lipids. The role of bacterial and archaeal membranes in adapting to environmental extremes has been reviewed in recent years $[42,43]$. Enzymes produced by the cold-adapted microorganisms are known to have higher activities at lower temperatures in comparison with their mesophilic and thermophilic counterparts [24]. Bioprospection of microorganisms from any ecological niche needs three major components including taxonomic as well as genetic diversity, the ability to produce biomolecules of biotechnological applications, and establishment of a facility for preservation/conservation of the microbial cultures. In bioprospection perspective of these psychrotolerant bacteria, their preservation in Culture Collection will help in advanced studies on these bacterial cultures in future.

\section{Conclusion}

The extreme cold environments restrict the normal life and are mainly inhabited by the cold-adapted microorganisms. Bioprospection of cold-adapted microorganisms, with their ability to produce cold-active biomolecules, is evolving as an interesting area in industrial biotechnology. The research groups are consistently trying to figure out the most abundant taxa of such microbial communities following various methods. With advancement in taxonomy, the phylogenetic methods based on the analysis of the 165 rDNA using DNA sequencing emerged and dominated as a reliable tool for species-level identification of bacteria.

New technologies for accurate and rapid identification of bacteria have always been desired. The emerging studies indicate that identification of microorganisms can now be achieved using protein 'fingerprints' measured by MALDI-TOF mass spectrometry. In this perspective, the polyphasic approach that has been considered as a combination of phenotypic and genotypic characters needs to be updated with inclusion of new technologies such as MALDI-TOF MS. However, due to difficulties with the reproducibility of results because of the use of different cultivation conditions and the limited availability of reference main spectra, MALDI-TOF MS-based biotyping has not yet been widely used for species identification. However, in view of the upcoming advanced and high-throughput methods along with the expansion of databases it is being recommended as a preferred tool for identification of microorganisms.

Acknowledgements The authors gratefully acknowledge the Director(s) of G.B. Pant National Institute of Himalayan Environment and Sustainable Development, Almora, and National Center for Cell Science, Pune, for extending the facilities.

Funding This study was funded by the Ministry of Environment, Forest and Climate Change, Government of India.

\section{Compliance with ethical standards}

Conflict of interest The authors declare that they have no conflict of interest.

Ethical approval This article does not contain any studies with human participants or animals performed by any of the authors.

\section{References}

1. Barria C, Malecki M, Arraiano CM (2013) Bacterial adaptation to cold. Microbiology 159:2437-2443 
2. Barroca M, Santos G, Gerday C, Collins T (2017) Biotechnological aspects of cold-active enzymes. In: Margesin R (ed) Psychrophiles: from biodiversity to biotechnology. Springer, Cham

3. Brooke JS (2012) Stentrophomonas maltophilia: an emerging global opportunistic pathogen. Clin Microbiol Rev 25(1):2-41

4. Colwell RR, Grimes DJ (2000) Nonculturable microorganisms in the environment. ASM Press, Washington DC

5. Das S, Dash HR, Mangwani N, Chakraborty J, Kumari S (2014) Understanding molecular identification and polyphasic taxonomic approaches for genetic relatedness and phylogenetic relationships of microorganisms. J Microbiol Methods 103:80-100

6. De Bruyne K, Slabbinck B, Waegeman W, Vauterin P, Baets BD, Vandamme $P$ (2011) Bacterial species identification from MALDITOF mass spectra through data analysis and machine learning. Syst Appl Microbiol 34:20-29

7. Dhakar K, Pandey A (2016) Wide $\mathrm{pH}$ range tolerance in extremophiles: towards understanding an important phenomenon for future biotechnology. Appl Microbiol Biotechnol 100:2499-2510

8. Dumorné K, Córdova DC, Astorga-Eló M, Renganathan P (2017) Extremozymes: a potential source for industrial applications. J Microbiol Biotechnol 27(4):649-659

9. Edgar RC (2004) MUSCLE: multiple sequence alignment with high accuracy and high throughput. Nucleic Acids Res 32(5):1792-1797

10. Fenselau C, Demirev PA (2001) Characterization of intact microorganisms by MALDI mass spectrometry. Mass Spectrom Rev 20:157-171

11. Gangwar P, Alam SI, Bansod S, Singh L (2009) Bacterial diversity of soil samples from the western Himalayas, India. Can J Microbiol 55:564-577

12. Gekenidis M-T, Studer $P$, Wüthrich $S$, Brunisholz R, Drissner D (2014) Beyond the matrix-assisted laser desorption ionization (MALDI) biotyping workflow: in search of microorganism-specific tryptic peptides enabling discrimination of subspecies. Appl Environ Mirobiol. https://doi.org/10.1128/aem.00740-14

13. Harmsen D, Rothgänger J, Frosch M, Albert J (2002) RIDOM: ribosomal differentiation of medical micro-organisms database. Nucleic Acids Res 30:416-417

14. Hébraud M, Potier P (1999) Cold shock response and low temperature adaptation in psychrotrophic bacteria. Mol Microbiol Biotechnol 1(2):211-219

15. Holland RD, Wilkes JG, Rafii F, Sutherland JB, Persons CC, Voorhees KJ, Lay JOJ (1996) Rapid identification of intact whole bacteria based on spectral patterns using matrix-assisted laser desorption/ionization with time-of-flight mass spectrometry. Rapid Commun Mass Spectrom 10:1227-1232

16. Jain R, Pandey A (2016) A phenazine-1-carboxylic acid producing polyextremophilic Pseudomonas chlororaphis (MCC2693) strain, isolated from mountain ecosystem, possesses biocontrol and plant growth promotion abilities. Microbiol Res 190:63-71

17. Jain R, Pandey A, Pasupuleti M, Pande V (2017) Prolonged production and aggregation complexity of cold-active lipase from Pseudomonas proteolytica (GBPI_Hb61) isolated from cold desert Himalaya. Mol Biotechnol 59(1):34-45

18. Kaira GS, Dhakar K, Pandey A (2015) A psychrotolerant strain of Serratia marcescens (MTCC 4822) produces laccase at wide temperature and $\mathrm{pH}$ range. AMB Express 5(1):1

19. Koubek J, Uhlik O, Jecna K, Junkova P, Vrkoslavova J, Lipov J, Kurzawova V, Macek T, Mackova M (2012) Whole-cell MALDITOF: Rapid screening method in environmental microbiology. Int Biodeterior Biodegradation 69:82-86

20. Kumar S, Stecher G, Tamura K (2016) MEGA7: molecular evolutionary genetics analysis version 7.0 for bigger datasets. Mol Biol Evol 33:1870-1874
21. Kurli R, Chaudhari D, Pansare AN, Khairnar M, Shouche YS, Rahi P (2018) Cultivale microbial diversity associated with cellular phones. Front Microbiol 9:1229

22. Leisner JJ, Laursen BG, Prévost H, Drider D, Dalgaard P (2007) Carnobacterium: positive and negative effects in the environment and in foods. FEMS Microbiol Rev 31(5):592-613

23. Li H, Yu Y, Luo W, Zeng Y, Chen B (2009) Bacterial diversity in surface sediments from the Pacific Arctic Ocean. Extremophiles 13:233-246

24. Loperena L, Soria V, Varela $H$, Lupo $S$, Alejandro Bergalli $A$, Guigou M, Pellegrino A, Bernardo A, Calviño A, Rivas F, Batista S (2012) Extracellular enzymes produced by microorganisms isolated from maritime Antarctica. World J Microbiol Biotechnol 28:2249-2256

25. Mandic-Mulec I, Prosser Jl (2011) Diversity of endospore-forming bacteria in Soil: Characterization and driving mechanisms. In: Logan NA, De Vos P (eds) Endospore-forming soil bacteria, soil biology 27. Springer, Berlin, pp 31-59

26. Margesin R, Miteva V (2011) Diversity and ecology of psychrophilic microorganisms. Res Microbiol 162:346-361

27. Morita RY (1975) Psychrophilic bacteria. Bacteriol Rev 39(2):144-167

28. Murray PR (2012) What is new in clinical microbiology-microbial identification by MALDI-TOF mass spectrometry. J Mol Diagn 14(5):419-423

29. Okubo T, Tosaka $Y$, Sato $T$, Usui $M$, Nakajima $C$, Suzuki $Y$, Imura S, Tamura Y (2014) Bacterial diversity in sea ice from the Southern Ocean and the Sea of Okhotsk. J Appl Environ Microbiol 2(6):266-272

30. Pandey A, Dhakar K, Jain R, Pandey N, Gupta VK, Kooliyottil R, Dhyani A, Malviya MK, Adhikari P (2018) Cold adapted fungi from Indian Himalaya: untapped source for bioprospecting. Proc Nat Acad Sci India (Sect B) Biol Sci 5:5. https://doi.org/10.1007/ s40011-018-1002-0

31. Pandey A, Durgapal A, Joshi M, Palni LMS (1999) Influence of Pseudomonas corrugata inoculation on root colonization and growth promotion of two important hill crops. Microbiol Res 154:259-266

32. Pandey A, Yarzábal LA (2018) Bioprospecting cold-adapted plant growth promoting microorganisms from mountain environments. Appl Microbiol Biotechnol 103(2):643-657. https://doi. org/10.1007/s00253-018-9515-2

33. Pesciaroli C, Cupini F, Selbmann L, Barghini P, Fenice M (2012) Temperature preferences of bacteria isolated from seawater collected in Kandalaksha Bay, White Sea, Russia. Polar Biol 35:435-445

34. Rahi P, Prakash O, Shouche YS (2016) Matrix-assisted laser desorption/ionization time-of-flight mass-spectrometry (MALDITOF MS) based microbial identifications: challenges and scopes for microbial ecologists. Front Microbiol 7:1359

35. Raymond-Bouchard I, Tremblay J, Altshuler I, Greer CW, Whyte LG (2017) Comparative transcriptomics of cold growth and adaptive features of a eury- and steno-psychrophile. Front Microbiol 9:1565

36. Russell NJ (1990) Cold adaptation of microorganisms. Philos Trans R Soc Lond 326:595-611

37. Saitou N, Nei M (1987) The neighbor-joining method: a new method for reconstructing phylogenetic trees. Mol Biol Evol 4:406-425

38. Seckbach J, Oren A, Stan-Lotter H (2013) Polyextremophiles: life under multiple forms of stress. Springer, Dordrecht

39. Sharma A, Shouche S (2015) Microbial culture collection: national centre for cell science, Pune (Maharastra, India). ENVIS Newsl Himal Ecol 12(4):10

40. Shivaji S, Pratibha MS, Sailaja B, Kishore KH, Singh AK, Begum Z, Anarasi U, Prabagaran SR, Reddy GSN, Srinivas TNR (2011) 
Bacterial diversity of soil in the vicinity of Pindari glacier, Himalayan mountain ranges, India, using culturable bacteria and soil 16S rRNA gene clones. Extremophiles 15:1-22

41. Siddiqui KS, Williams TJ, Wilkins D, Yau S, Allen MA, Brown MV, Lauro FM, Cavicchioli R (2013) Psychrophiles. Annu Rev Earth Planet Sci 41:6.1-6.29

42. Siliakus MF, van der Oost J, Kengen SWM (2017) Adaptations of archaeal and bacterial membranes to variations in temperature, $\mathrm{pH}$ and pressure. Extremophiles 21:651-670

43. Singh AK, Sad K, Singh SK, Shivaji S (2014) Regulation of gene expression at low temperature: role of cold-inducible promoters. Microbiology 160:1291-1297

44. Strejcek M, Smrhova T, Junkova P, Uhlik O (2018) Whole-cell MALDI-TOF MS versus 16S rRNA gene analysis for identification and dereplication of recurrent bacterial isolates. Front Microbiol. https://doi.org/10.3389/fmicb.2018.01294

45. Timperio AM, Gorrasi S, Zolla L, Fenice M (2017) Evaluation of MALDI-TOF mass spectrometry and MALDI BioTyper in comparison to $16 \mathrm{~S}$ rDNA sequencing for the identification of bacteria isolated from Arctic sea water. PLoS ONE 12(7):e0181860

46. Torres MJ, Petroselli G, Daz M, Erra-Balsells R, Audisio MC (2015) Bacillus subtilis subsp. subtilis CBMDC3f with antimicrobial activity against Gram-positive food borne pathogenic bacteria: UVMALDI-TOF MS analysis of its bioactive compounds. World J Microbiol Biotechnol 31(6):929-940
47. Trivedi P, Pandey A, Sa TM (2007) Chromate reducing and plant growth promoting activities of psychrotrophic Rhodococcus erythropolis MTCC 7905. J Basic Microbiol 47:513-517

48. Wenzler E, Kamboj K, Balada-Llasat JM (2015) Severe Sepsis Secondary to Persistent Lysinibacillus sphaericus, Lysinibacillus fusiformis and Paenibacillus amylolyticus bacteremia. Int J Infect Dis 35:93-95

49. Yadav AN, Sachan SG, Verma P, Saxena AK (2015) Prospecting cold deserts of north western Himalayas for microbial diversity and plant growth promoting attributes. J Biosci Bioeng 119(6):683-693

50. Zdanowski MK, Zmuda-Baranowska MJ, Borsuk P, Swiatecki A, Gorniak D, Wolicka D, Jankowska KM, Grzesiak J (2013) Culturable bacteria community development in postglacial soils of Ecology Glacier, King George Island, Antarctica. Polar Biol 36:511-527

51. Zhao X, Zhong J, Wei C, Lin C, Ding T (2017) Current perspectives on viable but non culturable state in food borne pathogens. Front Microbiol 8:580

Publisher's Note Springer Nature remains neutral with regard to jurisdictional claims in published maps and institutional affiliations. 